\title{
LETTERS
}

\section{Globalising artificial intelligence for improved clinical practice}

Published online on November 9, 2019. DOI: 10.20529/ IJME.2019.073.

Artificial intelligence (Al) technologies are facilitating the work of modern healthcare organisations to leverage the power of big data in clinical practice (1). In most cases, Al-based systems improve clinical decision-making using multiple layers of information and pre-specified algorithms (2). In addition, recent $\mathrm{Al}$ technologies like machine learning can learn from existing data and perform predictive operations resulting in a robust performance in clinical settings $(1,2)$. Such innovations are likely to serve the healthcare industry by minimising human error, savings costs, and maximising informed decisionmaking (2). However, critical challenges may affect the applications of $\mathrm{Al}$ in clinical settings, which include the effects on patient-provider communication, safety and efficacy of health services, and humane aspects of caregiving $(1,2)$. These issues suggest the need for a more careful analysis of different ethical aspects before adopting $\mathrm{Al}$ in clinical practice.

Several agencies have started developing guidelines and regulatory frameworks for using $\mathrm{Al}$ in clinical practice, like, the High-Level Expert Group on Al of the European Commission, which has presented its "Ethics guidelines for trustworthy $\mathrm{Al}^{\prime}$ " which proposed that the development of $\mathrm{Al}$ should be lawful, ethical, and robust (3). This highlights the need for considering multi-dimensional aspects of Al, with potentially complex medico-legal and ethical implications. Moreover, Albased systems are continuously evolving, making it essential to maintain the balance between technological advancements and their safe use in clinical operations. A recent regulatory framework proposed by the US Food and Drug Administration acknowledges this issue, arguing that future modifications of Al-based technologies should emphasise safe and effective use (4). While such guidelines are essential for optimal development and implementation of Al-based clinical systems, most of them have local or regional scope rather than a global vision. In the era of continued globalisation, it is critical to recognise the pre-existing digital divide among global nations; how it can be aggravated by newer technologies like Al; and how future advancements should address these challenges.

Digital health technologies are increasingly being used to strengthen health systems in many low- and middleincome countries. However, very few of those technologies are applied in clinical settings in these countries. In such resource-constrained contexts, Al-based clinical systems are likely to arrive late and incur a high cost to the users or health systems. In addition, developing nations do not have adequate resources to pursue advanced research and development in such advanced technologies. Therefore, a digital divide continues to exist between the developed and developing nations.

Furthermore, clinical practice guidelines are diverse across contexts and populations. In this scenario, new guidelines for $\mathrm{Al}$ in different countries may add more complexities in clinical practice, globally. Interestingly, such crises can be prevented through the same Al technologies, which can be used to reduce complexities and improve clinical practice given an integration of $\mathrm{Al}$ in clinical settings under uniform guidelines all over the world. In this process, the Al-based systems will be exposed to diverse and big data essential for training and testing, yielding greater precision in clinical decision-making in different contexts. Moreover, the use of Al in integrating genomic, epigenetic, and behavioural data can better inform personalised diagnosis and treatment across populations (2). Al can also be used to analyse economic, political, and technological challenges in a population and inform clinical decision-making accordingly, which can help in achieving equality and sustainability in global health systems (1).

To unleash these opportunities, a global vision for developing and using $\mathrm{Al}$ in clinical practice is essential. It can be achieved by fostering collaboration between scholars and institutions across the globe with a focus on the developing countries, which have a more significant proportion of the global burden of diseases, along with a focus on capacity building. Without advancing medical education in the era of digital health, clinical practitioners may not achieve the competencies to serve within a technologically advanced healthcare system. Recent initiatives by the World Health Organization and the International Telecommunication Union for benchmarking Al in healthcare offer promise of improving Al-driven processes and outcomes (5). As procedures are being developed, adopting globalised approaches within these efforts may facilitate the overcoming of the existing digital health challenges and prevent future disparities in Al-based clinical practice.

Md Mahbub Hossain (mhossain@tamu.edu), School of Public Health, Texas A \& M University, Texas, USA; Rachit Sharma (rachit. sharma@somaarth.org), The INCLEN Trust International, Okhla Industrial Area Phase-1, New Delhi 110020 INDIA; Abida Sultana (abidamona21@gmail.com), Nature Study Society of Bangladesh, Khulna 9000, BANGLADESH; Samia Tasnim (tasnim@tamu.edu), School of Public Health, Texas A \& M University, Texas, USA; Farah Faizah (faizah@unfpa.org), United Nations Population Fund, IDB Bhaban, Dhaka 1207, BANGLADESH.

\section{References}

1. Jiang, $F$, Jiang $Y$, Zhi H, Dong $Y$, Li H, Ma S, Wang Y. Artificial intelligence in healthcare: past, present and future. Stroke Vasc Neurol. 2017 Jun 21; 2(4):230-43. 
2. Shahid N, Rappon T, Berta W. Applications of artificial neural networks in health care organizational decision-making: A scoping review. PloS One. 2019 Feb 19; 14(2): e0212356.

3. European Commission. Ethics guidelines for trustworthy Al | Digital Single Market. 2019 Apr 8[cited 2019 Oct 20]. Available from: https:// ec.europa.eu/digital-single-market/en/news/ethics-guidelinestrustworthy-ai.

4. Food and Drug Administration. Proposed Regulatory Framework for Modifications to Artificial Intelligence/Machine Learning ( $\mathrm{Al} / \mathrm{ML}$ )-Based Software as a Medical Device (SaMD)-Discussion Paper and Request for Feedback. 2019 [cited 2019 Oct 21]. Available from: https://www. fda.gov/downloads/medicaldevices/deviceregulationandguidance/ guidancedocuments/ucm514737.pdf.

5. International Telecommunication Union (ITU). ITU-WHO Workshop on Artificial intelligence for health. 2018 Sep 25 [cited 2019 Oct 21]. Available from: https://www.itu.int/en/ITU-T/Workshops-andSeminars/20180925/Pages/default.aspx.

\section{Ethics of electronic tagging of dementia patients}

Published online on November 30, 2019. DOI: 10.20529/ IJME.2019.078.

Dementia is one of the most common neuropsychiatric disorders seen in old age with accompanying memory loss, aggressive behaviour, sleep problems and wandering behaviour with confusion (1). Many patients with dementia may be all alone at home with a domestic help and no family caregiver for most of the day and may sometimes wander off from their homes. Hence, dementia care programmes insist that an identity card with the patient's key details be worn, in case the patient wanders away and is unable to return home or inform people about the location of their home.

A medical company in India has recently launched an electronic tagging device for cell phones, to help relatives track the whereabouts of dementia patients beyond a certain perimeter of the home address fed into the device.

We raise here some ethical dilemmas involved in the electronic tagging of dementia patients. These are:

- Weighing the safety of the individual against the restrictions on their liberty, we may argue that electronic tagging may keep the person safe from an accident, or from being robbed or attacked. Studies show tagging has helped to find wandering patients faster, besides allowing them greater mobility. It may help reduce the anxieties of caregivers in a home or nursing home setting. Respectfully, electronic tagging is like wearing a waist band and far better than a patient with dementia being restrained in a nursing home to prevent wandering (2).

- Who takes the decision to use electronic tagging and when it is to be installed? Does the first instance of wandering warrant tagging, or is it installed after repeating wanderings? There are no guidelines for this. When patients with dementia lack total capacity, the relatives make this decision but the dilemma arises when patients have partial decision-making capacity and need to be involved in such decisions. Is the decision taken in the patient's interest or that of peace of mind for the caregivers? Tagging may be the least restrictive remedy for wandering, but would it make the caregiver less vigilant knowing that the alarm would beep in case of their charge wandering away? (3)

- Some studies find that wandering within limits may provide exercise and an enhanced sense of independence to the individual (4). In light of recent positive trends in psychology, based on the concepts of personhood and citizenship, such devices may help in ensuring a better quality of life for people living with dementia (5).

In India, tagging may have specific social and cultural implications. People may feel that it is like tagging of criminals or animals. However, several patients with dementia in India are kept behind locked doors and a wandering episode can precipitate a patient's placement in residential care. Assistive technology, like e- tagging may facilitate the patient's remaining at home. On the other hand, 24-hour call centres will be needed for monitoring these devices and tracking them, using up the country's scarce resources (6).

- Patients with dementia need social interaction for a better quality of life, and dependence on electronic tagging may result in caregivers spending less time with patients. Clinicians and caregivers must note that tracking methods cannot replace quality care and meaningful relationships in patients with dementia (7).

- GPS tracking technology and personal information revealed by the linking of personal and Google accounts may be used by companies to electronically bombard caregivers with information about dementia care products and shelters and raise privacy concerns for patients and caregivers.

- A final issue is that while there are small anecdotal case studies and case series, no trials have been found for the efficacy of this technology. Though no safety issues have been noted so far, efficacy is difficult to establish reliably, unless randomised controlled trials are undertaken. After considering the pros and cons of this technology, it inevitably raises privacy and human rights concerns. However, it must be kept in mind that individuals using smart phones, often use "location services" for tracking their children or friends for safety during late night taxi rides or travelling to relatively unsafe places; or to provide the location of a particular place. Such use does not necessarily make caregivers lax.

This technology needs to be tested. In comparison to locked rooms, physical restraint and sedation, it is the least restrictive method of handling wandering behaviour (8) and further trials can provide information on real-time efficacy in order to understand its applications We also suggest that changing the terminology to replace the word "tagging" can also help to perceive the device as more acceptable and user-friendly.

Pragya Lodha,(pragya6lodha@gmail.cm), Independent Clinical Psychologist, Mumbai, Avinash De Sousa (corresponding author - avinashdes888@gmail.com), Research Associate, Department of 